\title{
Randomized, Controlled, Multi-center Trial: Comparing the Safety and Efficacy of DA-9701 and Itopride Hydrochloride in Patients With Functional Dyspepsia
}

\author{
Myung-Gyu Choi, ${ }^{1 *}$ Poong-Lyul Rhee, ${ }^{2}$ Hyojin Park, ${ }^{3}$ Oh Young Lee, ${ }^{4}$ Kwang Jae Lee, ${ }^{5}$ Suck Chei Choi, ${ }^{6}$ Sang Young Seol, ${ }^{7}$ \\ Hoon Jai Chun, ${ }^{8}$ Jong-Sun Rew, ${ }^{9}$ Dong Ho Lee, ${ }^{10}$ Geun Am Song, ${ }^{11}$ Hwoon Yong Jung, ${ }^{12}$ Hyung Yong Jeong, ${ }^{13}$ In Kyung Sung, ${ }^{14}$ \\ Joon Seong Lee, ${ }^{15}$ Soo Teik Lee, ${ }^{16}$ Sung Kook Kim, ${ }^{17}$ and Yong Woon Shin ${ }^{18}$ \\ ${ }^{1}$ Department of Internal Medicine, The Catholic University of Korea, Seoul, Korea; ${ }^{2}$ Sungkyunkwan University School of Medicine, Seoul, \\ Korea; ${ }^{3}$ Yonsei University College of Medicine, Seoul, Korea; ${ }^{4}$ Hanyang University College of Medicine, Seoul, Korea; ${ }^{5}$ Ajou University School \\ of Medicine, Suwon, Gyeonggi-do, Korea; ${ }^{6}$ Wonkwang University College of Medicine, Iksan, Jeollabuk-do, Korea; ' Inje University College of \\ Medicine, Busan, Korea; ${ }^{8}$ Korea University College of Medicine, Seoul, Korea; ${ }^{9}$ Chonnam National University College of Medicine, Gwangju, \\ Korea; ${ }^{10}$ Seoul National University College of Medicine, Seoul, Korea; ${ }^{11}$ Pusan National University College of Medicine, Busan, \\ Korea; ${ }^{12}$ University of Ulsan College of Medicine, Seoul, Korea; ${ }^{13}$ Chungnam National University College of Medicine, Daejeon, \\ Korea; ${ }^{14}$ Konkuk University College of Medicine, Seoul, Korea; ${ }^{15}$ Soonchunhyang University College of Medicine, Seoul, Korea; ${ }^{16}$ Chonbuk \\ National University College of Medicine, Jeonju, Jeollabuk-do, Korea; ${ }^{17}$ Kyungpook National University College of Medicine, Daegu, Korea; \\ and ${ }^{18}$ Inha University College of Medicine, Incheon, Korea
}

\section{Background/Aims}

Therapies of functional dyspepsia (FD) are limited. DA-9701 is a novel prokinetic agent formulated with Pharbitis semen and Corydalis Tuber. We aimed to assess the efficacy of DA-9701 compared with itopride in FD patients.

\section{Methods}

Patients with FD randomly received either itopride $50 \mathrm{mg}$ or DA-9701 $30 \mathrm{mg}$ t.i.d after a 2-week baseline period. After 4 weeks of treatment, 2 primary efficacy endpoints were analyzed: the change from baseline in composite score of the 8 dyspeptic symptoms and the overall treatment effect. Impact on patients' quality of life was assessed using the Nepean Dyspepsia Index (NDI) questionnaire.

Results

We randomly assigned 464 patients with 455 having outcome data. The difference of the composite score change of the 8 symptoms between the 2 groups was 0.62 , indicating that DA-9701 was not inferior to itopride. The overall treatment effect response rate was not different between the groups. When responder was defined as $\geq 5$ of the 7 Likert scale, responder rates were $37 \%$ of DA-9701 and 36\% of itopride group. Patients receiving DA-9701 experienced similar mean percentage of

Received: October 8, 2014 Revised: December 29, 2014 Accepted: January 7, 2015

(c) This is an Open Access article distributed under the terms of the Creative Commons Attribution Non-Commercial License (http://creativecommons. org/licenses/by-nc/4.0) which permits unrestricted non-commercial use, distribution, and reproduction in any medium, provided the original work is properly cited.

*Correspondence: Myung-Gyu Choi, MD, PhD

Division of Gastroenterology, Department of Internal Medicine, College of Medicine, The Catholic University of Korea, Seoul St. Mary's Hospital, 505 Banpodong, Seochogu, Seoul 137-701, Korea

Tel: +82-2-2258-6017, Fax: +82-2-2258-2089, E-mail: choim@catholic.ac.kr

Financial support: This study was supported by grant number PF06205-00 from the Plant Diverse Research Center of 21C Frontier R\&D Programs, Ministry of Science and Technology, Korea.

Conflicts of interest: None.

Author contributions: Myung-Gyu Choi wrote and performed the final editing of the manuscript. All of the authors contributed to the design of the study, subject recruitment and edited the manuscript.

ORCID: Myung-Gyu Choi, http://orcid.org/0000-0003-4083-5187. 
days with adequate relief during the 4-week treatment period compared with those receiving itopride (56.8\% vs $59.1 \%$ ). Both drugs increased the NDI score of 5 domains without any difference in change of the NDI score between the groups. The safety profile of both drugs was comparable.

\section{Conclusions}

DA-9701 significantly improves symptoms in patients with FD. DA-9701 showed non-inferior efficacy to itopride with comparable safety.

\section{(J Neurogastroenterol Motil 2015;21:414-422)}

\section{Key Words}

Clinical trial; DA-9701; Functional dyspepsia; Itopride

\section{Introduction}

Functional dyspepsia (FD) is a highly prevalent condition, ${ }^{1}$ and causes frequent health care utilization which results in increases in socioeconomic burden. FD is defined as the presence of symptoms thought to originate in the gastroduodenal region, in the absence of any organic, systemic, or metabolic disease that is likely to explain the symptoms. ${ }^{2}$ FD is not a life threatening condition, however, it could significantly impair the quality of life (QOL). ${ }^{3}$ The investigation of FD symptoms remains challenging and its pathophysiology may involve several mechanisms. The management of dyspepsia remains a major issue in clinical practice. Current treatment approaches to FD include gastric acid suppression and gastroprokinetic drugs as primary treatment options. However, satisfactory therapeutic approaches are not currently available in clinical practice. ${ }^{3}$ The role of Helicobacter pylori infection in FD has been controversial, ${ }^{5}$ although recent meta-analyses suggest a small benefit from $H$. pylori eradication. ${ }^{6}$ Antidepressant drugs may be considered in refractory or co-morbid cases, although the value of antidepressants in FD has not been established.

DA-9701 is a new prokinetic agent formulated from extracts of Pharbitidis semen and Corydalis tuber. ${ }^{7}$ These plants have been used in oriental traditional medicine for the treatment of gastrointestinal maladies. DA-9701 has been shown to improve gastric motility by normalizing delayed gastric emptying and enhancing gastric accommodation in animal models. ${ }^{7-10}$ The aim of this study was to assess the efficacy and safety of DA-9701 compared with itopride in patients with FD.

\section{Materials and Methods}

This was a multicenter, double-blind, randomized, controlled trial with concealed allocation comparing the safety and efficacy of DA-9701 (Motilitone $\left.{ }^{(}\right)$) and itopride hydrochloride. This study was performed in 18 institutions including The Catholic University of Korea Seoul St. Mary's hospital, Konkuk University Medical Center, Kyungpook National University Hospital, Korea University Medical Center, Pusan National University Hospital, Bundang Seoul National University Hospital, Samsung Medical Center, Soonchunhyang University Hospital, Asan Medical Center, Ajou Medical Center, Yonsei Univerisity Gangnam Severance Hospital, Wonkwang University of Medical and hospital, Inje University Pusan Paik Hospital, Inha University Hospital, Chonnam National University Hospital, Chonbuk National University Hospital, Chungnam National University Hospital and Hanyang University Medical Center. The trial was double blind; participants, investigators and site staff responsible for performing assessments remained blind from randomisation until database lock. It comprised a 2-week screening period, a 2-week observational baseline period, and a 4-week treatment period. Patients with FD were randomly assigned to receive either itopride $50 \mathrm{mg}$ or DA-9701 $30 \mathrm{mg}$ t.i.d after the 2-week observational baseline period.

\section{Patient Population}

The study subjects were patients $\geq 20$ years of age diagnosed with FD according to the modified Rome II criteria. ${ }^{11}$ Dyspeptic symptoms had to be present for the last 3 months with symptom onset at least 6 months prior to diagnosis. Patients should have had at least moderate symptom severity which was defined as bothersomeness score $\geq 3$ of 5 Likert scale of at least 
one dyspeptic symptom. All patients were required to have normal finding of upper gastrointestinal endoscopy. Key exclusion criteria included a history of gastrointestinal malignancies, erosive esophagitis, erosive gastroduodenitis, gastric or duodenal ulceration, concomitant serious medical conditions, current irritable bowel syndrome (IBS) or IBS-like symptoms, the use of nonsteroidal anti-inflammatory drugs, steroids or antisecretory or prokinetic medications, underlying psychiatric illness, a history of major abdominal surgery and patients in whom heartburn was the predominant symptom on the symptom list. Also, pregnant or breastfeeding women and those of childbearing age who were not using an approved method of contraception were excluded. Patients who were diagnosed with $H$. pylori infection by Campylobacter-like organism test were not excluded from the study.

\section{Trial Design}

The patients underwent a 14-day ( \pm 2 days) medication-free run-in phase. During the baseline observation period, patients who reported an average of at least "moderate severity" ( $\geq 3$ on a 5 -point Likert scale) for one or more of the 8 cardinal dyspepsia symptoms (upper abdominal pain, upper abdominal discomfort, epigastric burning, inability to finish regular meal, fullness after eating, pressure in upper abdomen, bloating, and nausea) were eligible for randomization. The patients were randomly allocated by a computerized random number table with the key code securely stored (Proc Plan procedure of SAS version 9.1; SAS Institute, Cary, NC, USA). The random number table was created by a block randomization method. Patients were randomized to either the DA-9701 or the itopride group. Subsequently, the patients received daily DA-9701 $30 \mathrm{mg}$ or itopride $50 \mathrm{mg}$ with the same shaped counterpart placebo 3 times a day for a period of 4 weeks. During the 2 -week observational baseline period and the 4-week treatment period, patients rated their dyspepsia symptoms on an 8 item symptom checklist. The symptom scale measured the bothersomeness of each symptom, applying a scale of, and 0 (not at all) to 4 (extremely bothersome). The composite scores were calculated by the sum of score of the 8 dyspeptic symptoms. The impact of study medication on patients' health related quality of life (HRQOL) was assessed using the Nepean Dyspepsia Index (NDI) questionnaire, a validated, disease-specific HRQOL measure. ${ }^{12,13}$ NDI comprises 25 items and 5 domains (tension, interference with daily activities, eating/drinking, knowledge/control, and work/study). Patients rated each item, recalling the previous 2 weeks, on a 5 -point Likert scale, provid- ing individual domain scores ranging from 1 to 100 . The NDI was administered at baseline, at week 2 of study medication, and at the end of treatment.

Previous validation study of the Korean version of NDI revealed an excellent test-retest reproducibility and internal consistency which was expressed by Cronbach's alpha, 0.92. ${ }^{11}$ Construct was validated by comparison with Short form-36 health survey, global rating of QOL, and anxiety and intrusiveness. Responsiveness of the NDI instrument was performed as part of the cross-cultural validation. ${ }^{14}$

On a daily basis, patients responded to a daily adequate relief question in the paper diaries. The question was "Have you had adequate relief of dyspeptic symptoms in the last day?" as assessed by the binary response (yes or no). After 4-week treatment period, patients completed an "overall treatment effect" (OTE) questionnaire. The question was "Please consider how you felt during the past treatment period in regard to your dyspepsia, in particular your overall well-being, and symptoms of dyspepsia? This was scored on a 7-point Likert scale ranging from complete relief to no response or aggravation.

\section{Efficacy Assessments}

The treatment effect was measured by 2 primary efficacy endpoints: (1) The change from baseline in composite score of the 8 dyspeptic symptoms. (2) OTE as rated on a 7-grade scale. Responder was defined as giving a response of "no symptoms," "a major response" or "quite bit a response" on the 7-point Likert scale.

Secondary measures of efficacy included mean percentage of days with adequate relief of dyspepsia symptoms, change of individual symptoms and the NDI QOL questionnaire. Treatment compliance was defined as the percentage of used tablets. Compliance over $80 \%$ was considered acceptable.

\section{Tolerability and Safety Assessments}

All adverse events and serious adverse events (a medically significant event that was life-threatening, required hospitalization, or caused significant incapacity) were recorded. In addition, vital signs, hematology, urinalysis, blood chemistry, and electrocardiograph data were evaluated. Patients were withdrawn from the trials if they interrupted study medication for $>5$ consecutive days, and reasons for patient dropout were recorded.

\section{Statistical Methods}

The SAS system version 9.1 was used to perform all efficacy 
and safety analyses. Target enrollment for trial was 468 (234 patients per trial). The margin of noninferiority was set as 1.7 from a previous placebo-controlled study (unpublished data) and represented less than $50 \%$ of the difference in the change from baseline in the composite score of the eight dyspeptic symptoms between two groups. The number of subjects was determined assuming a level of significance of $\alpha=0.025$ (one-sided) and an $80 \%$ statistical power of each test.

Demographic and baseline characteristics were summarized descriptively for all the participating patient subjects. For inter-group differences, a Shapiro-Wilk test was performed to testing for normality of the distribution and two-sample $t$ test was used to evaluate statistical significance of continuous data, and
Chi-squared test was used on categorical data.

Efficacy outcomes were analyzed in the full-analysis (FA) and per protocol population of all randomized patients. The FA population referred to the subjects group excluding those who have not once been administered with the investigational products or are not once evaluated for the primary efficacy endpoint after the administration of the investigational products. Adverse events were evaluated in the safety population, which was defined as all patients who received at least one dose of study medication.

For the primary efficacy endpoint of the change from baseline in composite score of the 8 dyspeptic symptoms, the upper limit of one-sided $97.5 \%$ confidence interval (CI) of difference between DA-9701 and itopride was computed. The change from

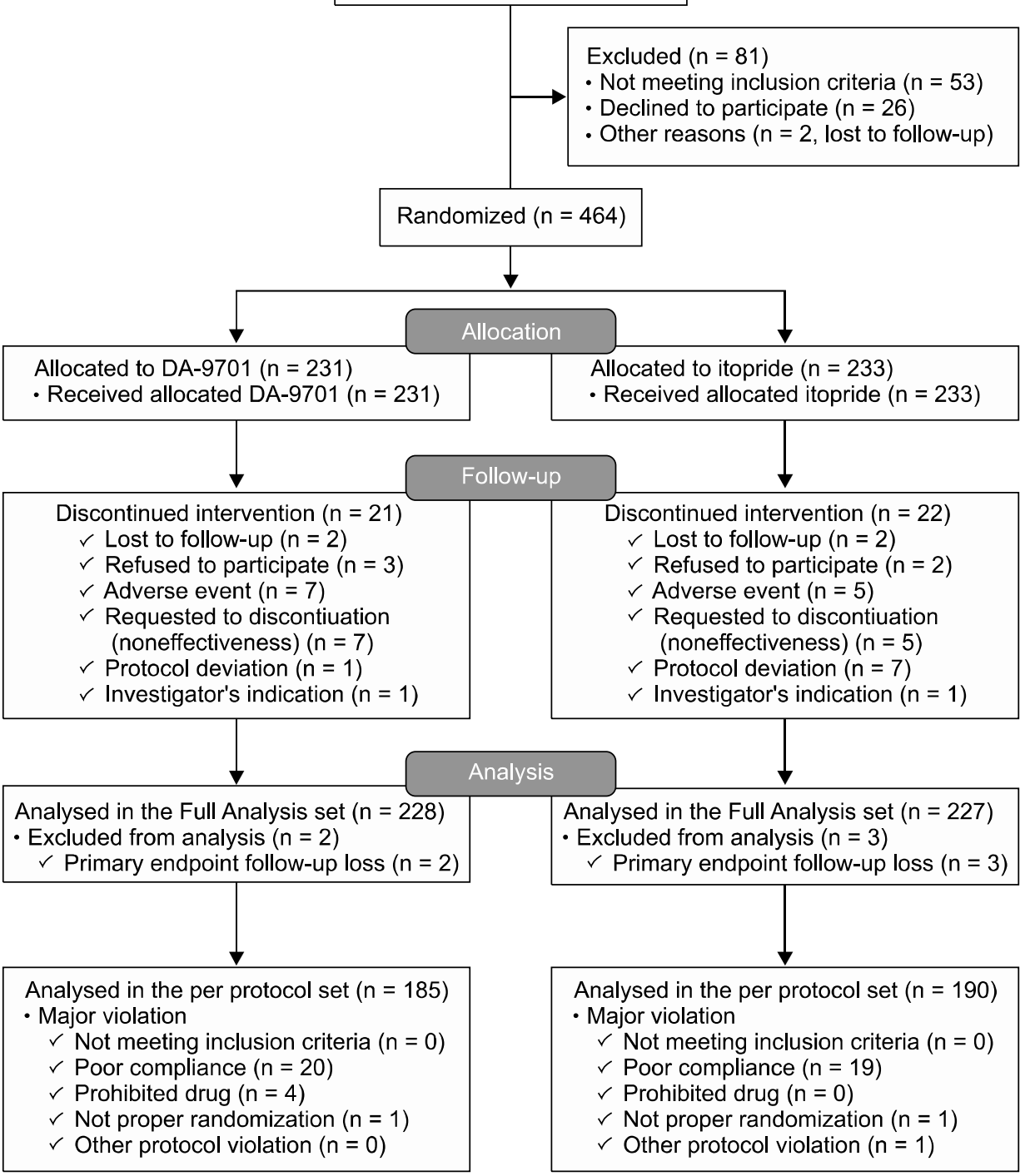

Figure. Summary of patient flow. Subjects who discontinued intervention or had a major violation were excluded from per protocol analysis. Some subjects had overlapping exclusion factors. 
baseline in composite score of the 8 dyspeptic symptoms efficacy of DA-9701 (test group) could be considered non-inferior to the itopride (control group) if the upper limit of the one-sided 97.5\% CI was equal to or less than 1.7 as noninferiority margin. A frequency distribution table was prepared for the OTE at the last survey point and thereby the improvement rates were determined. The difference of OTE and responder rates between the two treatments groups were compared statistically by the Chisquared test. Mean percentage of days with adequate relief of dyspepsia symptoms, change of individual symptoms, and the NDI QOL questionnaire were measured as secondary efficacy variables of the study. For the mean percentage of days with adequate relief of dyspepsia symptoms, change of individual symptoms and the NDI quality of life questionnaire, the intergroup differences were analyzed using two-sample Student's $t$ test under the assumption of normality.

For adverse events, 95\% confidence interval for the frequency $(\%)$ of occurrence of adverse events were calculated for subjects who experienced one or more cases of adverse drug reaction. Inter-group comparisons were conducted using Chisquared test.

The study protocols were approved by the ethics committees at all participating sites and were performed in compliance with the Declaration of Helsinki and the Good Clinical Practice implemented in Japan. Written informed consent was obtained from every patient.

\section{Results}

\section{Patient Characteristics}

A total of 545 patients were screened, and finally 464 patients were randomly allocated (Figure). The most frequent reasons for exclusion prior to randomization were organic diseases, co-morbidity and dyspepsia symptoms that did not meet the severity criteria during the baseline period. Nine percent of patients in the DA-9701 (21/231) and itopride (22/233) groups did not complete the study. Four hundred and fifty-five patients were avail-

Table 1. Patient Baseline Demographic Characteristics

\begin{tabular}{lccc}
\hline & DA-9701 $(\mathrm{n}=231)$ & Itopride $(\mathrm{n}=233)$ & $P$-value \\
\cline { 2 - 3 } & \multicolumn{2}{c}{ Patients $(\%)$ or Mean $\pm \mathrm{SD}$} & 0.4125 \\
Age $(\mathrm{yr})$ & $41.35 \pm 13.43$ & $40.31 \pm 14.03$ & 0.6575 \\
Female $(\%)$ & $163(70.6)$ & $160(68.7)$ & 0.8750 \\
Height $(\mathrm{cm})$ & $162.84 \pm 8.03$ & $162.95 \pm 7.35$ & 0.7940 \\
Weight $(\mathrm{kg})$ & $59.41 \pm 10.76$ & $59.15 \pm 10.97$ & 0.6604 \\
Body mass index $\left(\mathrm{kg} / \mathrm{m}^{2}\right)$ & $22.3 \pm 3.0$ & $22.2 \pm 3.2$ & 0.8504 \\
Smoking & $22(9.5)$ & $25(10.7)$ & 0.1952 \\
Alcohol Drinking & $74(32.0)$ & $88(37.8)$ & 0.7867 \\
Total dyspeptic symptoms & $12.70 \pm 5.40$ & $13.10 \pm 5.80$ & \\
Composite score & $101 / 229(44.1 \%)$ & $92 / 232(39.6 \%)$ & \\
Helicobacter pylori $(+)$ & & & \\
\hline
\end{tabular}

${ }^{\mathrm{a}}$ The composite scores were calculated from the sum of the bothersomeness scores of the 8 dyspeptic symptoms.

Table 2. The Overall Treatment Effect by Patients

\begin{tabular}{lccccc}
\hline & \multicolumn{2}{c}{ Full analysis } & & \multicolumn{2}{c}{ Per protocol analysis } \\
\cline { 2 - 3 } \cline { 5 - 5 } & DA-9701 $(\mathrm{n}=227)$ & Itopride $(\mathrm{n}=226)$ & & DA-9701 (n=185) & Itopride $(\mathrm{n}=190)$ \\
\hline No response or aggravation & $21(9.3 \%)$ & $19(8.4 \%)$ & $8(4.3 \%)$ & $8(4.2 \%)$ \\
Slight response & $16(7.1 \%)$ & $28(12.4 \%)$ & & $14(7.6 \%)$ & $23(12.1 \%)$ \\
A little bit of a response & $56(24.7 \%)$ & $40(17.7 \%)$ & $43(23.2 \%)$ & $34(17.9 \%)$ \\
A moderate response & $51(22.5 \%)$ & $58(25.7 \%)$ & $44(23.8 \%)$ & $54(28.4 \%)$ \\
Quite a bit a response & $50(22.0 \%)$ & $47(20.8 \%)$ & & $44(23.8 \%)$ & $41(21.6 \%)$ \\
A major response & $27(11.9 \%)$ & $31(13.7 \%)$ & & $26(14.1 \%)$ & $27(14.2 \%)$ \\
No symptoms & $6(2.6 \%)$ & $3(1.3 \%)$ & $6(3.2 \%)$ & $3(1.6 \%)$ \\
\hline
\end{tabular}

Data are presented as number $(\%)$. Two patients, one in each group did not have overall treatment effect analyzed and were excluded from the full analysis. 
able for the FA analysis: 228 for DA-9701 versus 227 for itopride. Data on 375 patients were available for the per protocol analysis: 185 for DA-9701 versus 190 for itopride. Patients randomized to DA-9701 or itopride had similar demography, body mass index, and symptom scores at the baseline (Table 1).

\section{Primary Efficacy Variables}

The changes from baseline in composite score of the 8 dyspeptic symptoms were -6.89 in the DA-9701 group and -7.51 in the itopride group, respectively. The difference of the change from baseline in composite score of the 8 dyspeptic symptoms between the 2 groups was 0.62 . The upper limit of one-sided $97.5 \%$ $\mathrm{CI}$ is 1.54 , which is less than 1.7 , indicating that DA-9701 is not inferior to itopride. The response rate by the patient's OTE was not significantly different between the 2 groups. Patients who were with no response or aggravation, slight response or a little bit of a response and a moderate response indicated non-responders. Patients who were with quite a bit a response, a major response

Table 3. Change of Individual Symptom Score From Baseline

\begin{tabular}{|c|c|c|c|c|c|c|c|c|}
\hline \multirow{2}{*}{ FA set } & \multicolumn{4}{|c|}{ DA-9701 } & \multicolumn{4}{|c|}{ Itopride } \\
\hline & Baseline & 2 weeks & 4 weeks & Change & Baseline & 2 weeks & 4 weeks & Change \\
\hline Pain in upper abdomen & $1.37 \pm 0.09$ & $0.85 \pm 0.07$ & $0.62 \pm 0.06$ & $-0.76 \pm 0.09$ & $1.24 \pm 0.09$ & $0.78 \pm 0.07$ & $0.53 \pm 0.06$ & $-0.71 \pm 0.08$ \\
\hline $\begin{array}{l}\text { Discomfort in upper } \\
\text { abdomen }\end{array}$ & $1.97 \pm 0.08$ & $1.21 \pm 0.07$ & $0.93 \pm 0.07$ & $-1.04 \pm 0.08$ & $1.95 \pm 0.08$ & $1.19 \pm 0.07$ & $0.89 \pm 0.07$ & $-1.06 \pm 0.08$ \\
\hline Burning in upper abdomen & $1.45 \pm 0.09$ & $0.86 \pm 0.07$ & $0.64 \pm 0.06$ & $-0.82 \pm 0.09$ & $1.54 \pm 0.09$ & $0.93 \pm 0.07$ & $0.66 \pm 0.06$ & $-0.88 \pm 0.09$ \\
\hline Heartburn & $0.54 \pm 0.06$ & $0.43 \pm 0.05$ & $0.27 \pm 0.05$ & $-0.27 \pm 0.06$ & $0.69 \pm 0.07$ & $0.44 \pm 0.06$ & $0.30 \pm 0.05$ & $-0.40 \pm 0.08$ \\
\hline Cramps in upper abdomen & $0.52 \pm 0.07$ & $0.24 \pm 0.04$ & $0.18 \pm 0.03$ & $-0.34 \pm 0.07$ & $0.44 \pm 0.06$ & $0.33 \pm 0.05$ & $0.19 \pm 0.04$ & $-0.26 \pm 0.07$ \\
\hline Chest pain & $0.62 \pm 0.07$ & $0.36 \pm 0.05$ & $0.26 \pm 0.04$ & $-0.36 \pm 0.06$ & $0.72 \pm 0.07$ & $0.51 \pm 0.06$ & $0.25 \pm 0.04$ & $-0.48 \pm 0.07$ \\
\hline $\begin{array}{l}\text { Inability to finish } \\
\text { regular meal }\end{array}$ & $1.32 \pm 0.09$ & $0.96 \pm 0.07$ & $0.78 \pm 0.07$ & $-0.55 \pm 0.08$ & $1.44 \pm 0.09$ & $0.94 \pm 0.07$ & $0.72 \pm 0.07$ & $-0.71 \pm 0.09$ \\
\hline $\begin{array}{l}\text { Bitter tasting fluid that } \\
\text { comes to your mouth }\end{array}$ & $1.17 \pm 0.08$ & $0.75 \pm 0.06$ & $0.54 \pm 0.06$ & $-0.64 \pm 0.08$ & $1.26 \pm 0.09$ & $0.71 \pm 0.07$ & $0.39 \pm 0.05$ & $-0.88 \pm 0.09$ \\
\hline Fullness after eating & $2.43 \pm 0.08$ & $1.63 \pm 0.07$ & $1.16 \pm 0.07$ & $-1.27 \pm 0.09$ & $2.54 \pm 0.07$ & $1.43 \pm 0.07$ & $0.98 \pm 0.07$ & $-1.56 \pm 0.08$ \\
\hline Pressure in upper abdomen & $1.14 \pm 0.08$ & $0.63 \pm 0.07$ & $0.46 \pm 0.06$ & $-0.68 \pm 0.09$ & $1.16 \pm 0.09$ & $0.66 \pm 0.07$ & $0.44 \pm 0.06$ & $-0.71 \pm 0.09$ \\
\hline Bloating in upper abdomen & $2.12 \pm 0.08$ & $1.27 \pm 0.07$ & $1.00 \pm 0.07$ & $-1.12 \pm 0.09$ & $2.25 \pm 0.08$ & $1.37 \pm 0.08$ & $0.89 \pm 0.07$ & $-1.35 \pm 0.09$ \\
\hline Nausea & $0.85 \pm 0.08$ & $0.44 \pm 0.05$ & $0.32 \pm 0.05$ & $-0.54 \pm 0.08$ & $0.96 \pm 0.08$ & $0.44 \pm 0.06$ & $0.31 \pm 0.05$ & $-0.64 \pm 0.08$ \\
\hline Belching & $1.48 \pm 0.09$ & $1.08 \pm 0.07$ & $0.80 \pm 0.07$ & $-0.69 \pm 0.08$ & $1.60 \pm 0.09$ & $1.07 \pm 0.07$ & $0.71 \pm 0.06$ & $-0.89 \pm 0.09$ \\
\hline Vomiting & $0.35 \pm 0.06$ & $0.16 \pm 0.04$ & $0.13 \pm 0.03$ & $-0.23 \pm 0.05$ & $0.36 \pm 0.05$ & $0.17 \pm 0.04$ & $0.11 \pm 0.03$ & $-0.24 \pm 0.06$ \\
\hline Bad breath & $0.48 \pm 0.06$ & $0.33 \pm 0.05$ & $0.26 \pm 0.04$ & $-0.22 \pm 0.05$ & $0.71 \pm 0.07$ & $0.4 \pm 0.05$ & $0.24 \pm 0.04$ & $-0.48 \pm 0.07$ \\
\hline \multirow{2}{*}{$\mathrm{PP}$ set } & \multicolumn{4}{|c|}{ DA-9701 } & \multicolumn{4}{|c|}{ Itopride } \\
\hline & Baseline & 2 weeks & 4 weeks & Change & Baseline & 2 weeks & 4 weeks & Change \\
\hline Pain in upper abdomen & $1.34 \pm 0.1$ & $0.75 \pm 0.08$ & $0.49 \pm 0.06$ & $-0.84 \pm 0.09$ & $1.14 \pm 0.09$ & $0.65 \pm 0.07$ & $0.40 \pm 0.06$ & $-0.74 \pm 0.09$ \\
\hline $\begin{array}{l}\text { Discomfort in upper } \\
\text { abdomen }\end{array}$ & $1.94 \pm 0.09$ & $1.10 \pm 0.08$ & $0.83 \pm 0.07$ & $-1.11 \pm 0.09$ & $1.97 \pm 0.09$ & $1.10 \pm 0.08$ & $0.80 \pm 0.07$ & $-1.17 \pm 0.09$ \\
\hline Burning in upper abdomen & $1.40 \pm 0.10$ & $0.76 \pm 0.07$ & $0.47 \pm 0.05$ & $-0.93 \pm 0.09$ & $1.58 \pm 0.10$ & $0.88 \pm 0.07$ & $0.55 \pm 0.06$ & $-1.04 \pm 0.09$ \\
\hline Heartburn & $0.51 \pm 0.07$ & $0.39 \pm 0.06$ & $0.20 \pm 0.04$ & $-0.31 \pm 0.07$ & $0.69 \pm 0.08$ & $0.41 \pm 0.06$ & $0.23 \pm 0.04$ & $-0.47 \pm 0.08$ \\
\hline Cramps in upper abdomen & $0.50 \pm 0.07$ & $0.22 \pm 0.04$ & $0.15 \pm 0.04$ & $-0.35 \pm 0.08$ & $0.42 \pm 0.07$ & $0.27 \pm 0.05$ & $0.13 \pm 0.03$ & $-0.29 \pm 0.07$ \\
\hline Chest pain & $0.57 \pm 0.08$ & $0.31 \pm 0.05$ & $0.21 \pm 0.04$ & $-0.36 \pm 0.07$ & $0.73 \pm 0.08$ & $0.47 \pm 0.06$ & $0.20 \pm 0.04$ & $-0.53 \pm 0.08$ \\
\hline $\begin{array}{l}\text { Inability to finish regular } \\
\text { meal }\end{array}$ & $1.32 \pm 0.10$ & $0.93 \pm 0.08$ & $0.73 \pm 0.07$ & $-0.59 \pm 0.09$ & $1.43 \pm 0.10$ & $0.95 \pm 0.08$ & $0.69 \pm 0.07$ & $-0.74 \pm 0.10$ \\
\hline $\begin{array}{l}\text { Bitter tasting fluid that } \\
\text { comes to your mouth }\end{array}$ & $1.20 \pm 0.09$ & $0.77 \pm 0.07$ & $0.50 \pm 0.06$ & $-0.70 \pm 0.09$ & $1.31 \pm 0.10$ & $0.67 \pm 0.07$ & $0.31 \pm 0.05$ & $-1.00 \pm 0.09$ \\
\hline Fullness after eating & $2.45 \pm 0.08$ & $1.60 \pm 0.08$ & $1.11 \pm 0.08$ & $-1.35 \pm 0.10$ & $2.57 \pm 0.08$ & $1.41 \pm 0.08$ & $0.90 \pm 0.07$ & $-1.67 \pm 0.09$ \\
\hline Pressure in upper abdomen & $1.14 \pm 0.09$ & $0.57 \pm 0.07$ & $0.42 \pm 0.06$ & $-0.72 \pm 0.10$ & $1.13 \pm 0.09$ & $0.61 \pm 0.07$ & $0.39 \pm 0.06$ & $-0.73 \pm 0.10$ \\
\hline Bloating in upper abdomen & $2.11 \pm 0.09$ & $1.23 \pm 0.08$ & $0.94 \pm 0.08$ & $-1.17 \pm 0.10$ & $2.22 \pm 0.09$ & $1.32 \pm 0.08$ & $0.82 \pm 0.07$ & $-1.40 \pm 0.10$ \\
\hline Nausea & $0.81 \pm 0.08$ & $0.42 \pm 0.05$ & $0.29 \pm 0.05$ & $-0.52 \pm 0.09$ & $0.97 \pm 0.09$ & $0.42 \pm 0.06$ & $0.27 \pm 0.05$ & $-0.70 \pm 0.08$ \\
\hline Belching & $1.49 \pm 0.10$ & $1.10 \pm 0.08$ & $0.74 \pm 0.07$ & $-0.75 \pm 0.09$ & $1.57 \pm 0.10$ & $1.07 \pm 0.08$ & $0.66 \pm 0.06$ & $-0.91 \pm 0.09$ \\
\hline Vomiting & $0.32 \pm 0.06$ & $0.11 \pm 0.03$ & $0.09 \pm 0.03$ & $-0.23 \pm 0.06$ & $0.39 \pm 0.06$ & $0.14 \pm 0.04$ & $0.10 \pm 0.03$ & $-0.29 \pm 0.06$ \\
\hline Bad breath & $0.43 \pm 0.06$ & $0.31 \pm 0.05$ & $0.22 \pm 0.05$ & $-0.21 \pm 0.06$ & $0.71 \pm 0.08$ & $0.36 \pm 0.06$ & $0.21 \pm 0.04$ & $-0.50 \pm 0.08$ \\
\hline
\end{tabular}

Data are presented as mean $\pm \mathrm{SE}$. 
Table 4. Change of Individual Symptom Score From Baseline

\begin{tabular}{|c|c|c|c|c|c|c|c|c|}
\hline \multirow{2}{*}{ FA set } & \multicolumn{4}{|c|}{ DA-9701 } & \multicolumn{4}{|c|}{ Itopride } \\
\hline & Baseline & 2 weeks & 4 weeks & Change & Baseline & 2 weeks & 4 weeks & Change \\
\hline Tension/Sleep & $57.24 \pm 1.07$ & $65.03 \pm 1.15$ & $68.68 \pm 1.24$ & $11.44 \pm 1.33$ & $56.69 \pm 1.14$ & $67.66 \pm 1.19$ & $69.4 \pm 1.25$ & $12.72 \pm 1.21$ \\
\hline $\begin{array}{l}\text { Interference with daily } \\
\text { activities }\end{array}$ & $53.78 \pm 100$ & $59.93 \pm 1.09$ & $64.27 \pm 1.18$ & $10.49 \pm 1.12$ & $52.84 \pm 1.07$ & $61.97 \pm 1.14$ & $63.89 \pm 1.15$ & $11.05 \pm 1.2$ \\
\hline Eating/Drinking & $57.20 \pm 1.12$ & $64.30 \pm 1.24$ & $67.70 \pm 1.30$ & $10.50 \pm 1.33$ & $57.51 \pm 1.18$ & $64.45 \pm 1.24$ & $67.57 \pm 1.24$ & $10.05 \pm 1.45$ \\
\hline Knowledge/Control & $47.04 \pm 0.99$ & $56.08 \pm 1.16$ & $60.85 \pm 1.21$ & $13.81 \pm 1.20$ & $46.49 \pm 1.00$ & $57.47 \pm 1.11$ & $61.49 \pm 1.21$ & $15.00 \pm 1.18$ \\
\hline Work/Study & $56.09 \pm 1.12$ & $62.80 \pm 1.21$ & $66.51 \pm 1.23$ & $10.42 \pm 1.36$ & $57.40 \pm 1.17$ & $67.05 \pm 1.25$ & $67.87 \pm 1.25$ & $10.46 \pm 1.33$ \\
\hline \multirow{2}{*}{ PP set } & \multicolumn{4}{|c|}{ DA-9701 } & \multicolumn{4}{|c|}{ Itopride } \\
\hline & Baseline & 2 weeks & 4 weeks & Change & Baseline & 2 weeks & 4 weeks & Change \\
\hline Tension/Sleep & $56.65 \pm 1.16$ & $65.56 \pm 1.22$ & $69.56 \pm 1.32$ & $12.91 \pm 1.43$ & $56.79 \pm 1.23$ & $69.02 \pm 1.24$ & $70.67 \pm 1.31$ & $13.87 \pm 1.34$ \\
\hline $\begin{array}{l}\text { Interference with daily } \\
\text { activities }\end{array}$ & $54.04 \pm 1.08$ & $60.11 \pm 1.17$ & $65.20 \pm 1.27$ & $11.16 \pm 1.22$ & $52.92 \pm 1.10$ & $62.84 \pm 1.20$ & $65.03 \pm 1.22$ & $12.12 \pm 1.29$ \\
\hline Eating/Drinking & $57.21 \pm 1.24$ & $64.28 \pm 1.34$ & $68.04 \pm 1.37$ & $10.83 \pm 1.49$ & $57.36 \pm 1.29$ & $65.29 \pm 1.31$ & $68.46 \pm 1.31$ & $11.10 \pm 1.61$ \\
\hline Knowledge/Control & $47.17 \pm 1.06$ & $56.26 \pm 1.24$ & $61.58 \pm 1.28$ & $14.40 \pm 1.31$ & $46.94 \pm 1.10$ & $58.71 \pm 1.20$ & $63.03 \pm 1.29$ & $16.10 \pm 1.30$ \\
\hline Work/Study & $56.02 \pm 1.24$ & $62.94 \pm 1.29$ & $67.12 \pm 1.31$ & $11.10 \pm 1.50$ & $57.65 \pm 1.27$ & $68.19 \pm 1.33$ & $68.99 \pm 1.31$ & $11.35 \pm 1.42$ \\
\hline
\end{tabular}

Data are presented as mean $\pm \mathrm{SE}$.

Table 5. Incidence of Adverse Drug Events ( $>1 \%$ in Any Group)

\begin{tabular}{llc}
\hline \multirow{1}{*}{ Preferred Term } & $\begin{array}{l}\text { DA-9701 } \\
(\mathrm{n}=231)\end{array}$ & $\begin{array}{c}\text { Itopride } \\
(\mathrm{n}=233)\end{array}$ \\
\cline { 2 - 3 } & \multicolumn{2}{c}{ Number of patients $(\%)$} \\
\hline Constipation & $5(2.2)$ & $1(0.4)$ \\
Diarrhea & $4(1.7)$ & $8(3.5)$ \\
Alanine aminotransferase increased & $3(1.3)$ & $0(0.0)$ \\
Blood prolactin increased & $4(1.7)$ & $2(0.9)$ \\
Pruritus & $3(1.3)$ & $0(0.0)$ \\
\hline
\end{tabular}

and no symptoms were responders. In FA set analysis, $36.5 \%$ (83/227) of patients in the DA-9701 group and 35.8\% (81/226) of patients in the itopride group were responders. Patient's OTE was significantly correlated with the change of dyspeptic symptoms score from baseline (Table 2).

\section{Secondary Efficacy Variables}

Patients receiving DA-9701 experienced similar mean percentage of days with adequately symptom relief during the 4-week treatment period compared with those receiving itopride $(56.8 \pm 1.9$ vs $59.1 \pm 1.9)$. DA-9701 and itopride significantly reduced the score of all individual symptoms of dyspepsia from baseline (Table 3).

All NDI score of 5 domains at the baseline are low around
50 , which indicate poor quality of life in patients with FD. The score of the Knowledge/Control domain was lowest at baseline and showed the greatest improvement after treatment. Both of the drugs increased all NDI score indicating that patients' symptoms improved as well as quality of life. However, there was no difference in change in the NDI score of all five domains between the two groups (Table 4).

\section{Treatment Exposure, Safety, and Tolerability}

The incidence of adverse drug reactions was $8.7 \%$ in the DA-9701 group and $7.8 \%$ in the itopride group. No significant difference was seen between the 2 groups. The most commonly reported adverse events in both groups were constipation, headache, diarrhea, and increased prolactin level (Table 5). Pregnancy occurred in a patient in the DA-9701 group (0.4\%), and abortion in 2 patients and syncope in a patient in the itopride group were reported in the study. However, they were not related to trial drugs.

\section{Discussion}

Our study demonstrated that the new agent DA-9701 significantly improves symptoms and HRQOL in patients with FD. DA-9701 showed non-inferior efficacy to itopride and comparable safety profile.

DA-9701 is an agent obtained from the extracts of 2 plants 
which have been used in oriental traditional medicine for the treatment of gastrointestinal maladies. Pharbitidis Semen, the seed of Pharbitis nil Choisy, is known to have analgesic effects on abdominal disorders and induce diarrhea. ${ }^{15}$ Corydalis Tuber, the root of Corydalis yahusuo, has been used as a folk medicine for its analgesic and anti-ulcer effects. Previous animal studies suggest that DA-9701 has a variety of effects on gastrointestinal motor function and analgesic action. DA-9701 significantly accelerated gastric emptying caused by drugs and stress-induced animal models. DA-9701 increased gastric volume in a dose-dependent manner and improved gastric accommodation by increasing the postprandial gastric volume in a canine model. ${ }^{9}$ In a rat model of colorectal distension, DA-9701 may decrease visceral pain via the downregulation of phosphorylated extracellular signal-regulated kinase in the dorsal root ganglion and spinal cord. ${ }^{16}$ An in vitro study showed that DA-9701 has antagonistic effects on D2 and agonistic effects on 5-hydroxytryptamine 4 receptor $\left(5-\mathrm{HT}_{4}\right)$, 5- $\mathrm{HT}_{1 \mathrm{~A}}$, and 5- $\mathrm{HT}_{1 \mathrm{~B}}$ receptors. ${ }^{8,9,17,18}$ Electrical field stimulation on gastric fundus muscle strips demonstrate that the effect of DA-9701 on the rat gastric fundus relaxation is mainly mediated by nitrergic pathway. ${ }^{19}$

DA-9701 might be advantageous as a treatment option for FD due to its complex composition of herbal extracts, which act differently on multiple sites. It is likely that the mechanisms responsible for symptoms in functional gastrointestinal disorders may differ from one patient to another, and a single target may not achieve adequate efficacy in a patient population. Therefore, single receptor modulating drugs may be less likely to achieve a substantial therapeutic gain, if patients are not selected on the basis of the specific disorder. ${ }^{20}$ DA-9701 targets multiple pathophysiologic mechanisms contributing to the functional disorder rather than only an individual part or a specific receptor. Thus, this nonselective agent modulating multiple targets of the whole pathophysiologic process would be advantageous over highly selective medications addressing a single mechanism.

There is great concern about the safety issues of prokinetics in view of FD being of a benign nature, since several agents such as cisapride and tegaserod have been withdrawn due to side effects. DA-9701 was well tolerated and did not show drug related serious adverse events. Hyperprolactinemia has been reported in some patients receiving DA-9701 related to its anti-dopaminergic activity but this returned to normal after stopping it. Though our short-term study did not report any side effects, monitoring should be done during long-term use. The safety profile has an advantage, as herbal products are known to have less side effects. ${ }^{21}$ In this trial, we compared the efficacy of DA-9701 to itopride rather than placebo since the Korean FDA policy for the approval of a new drug allows comparison with a previously approved effective drug in the phase III trial. The efficacy of itopride for FD patients was controversial in Caucasian patients as recent clinical trials of itopride in the West have proved its efficacy over placebo. ${ }^{22}$ However, itopride obtained an approval for treatment of FD from Korean FDA several decades ago and was also widely approved in some Asian countries. Therefore, further placebo-controlled clinical trials of DA-9701 are needed. It has been difficult to establish consistent effects of drugs to treat FD symptoms in different trials in different parts of the world. Challenges in trials include optimal patient selection and the lack of established and generally accepted endpoints for efficacy evaluation. ${ }^{23}$

The optimal endpoint to be used in FD clinical trials has not been established. ${ }^{23}$ The change from baseline in composite score of the 8 dyspeptic symptoms was the primary efficacy endpoint. We also used the OTE as a primary endpoint which has the advantage of using a patient-derived global symptomatic evaluation, as recommended by the Rome guidelines. ${ }^{24}$ This method accurately provides the magnitude of response and overcomes some of the limitations of a binary endpoint. The OTE was used most recently in the acotiamide trial as a primary endpoint ${ }^{25}$ and the tegaserod trial as a secondary endpoint. ${ }^{26}$ The seven point Likert scale of OTE in both trials allocated 3 scales for measuring the degree of aggravation from slight to extreme. However, only few patients were reported as being aggravated. This effectively turned the 7-point scale into a 4-point one, whereas our 7-point scale seems to provide greater sensitivity and reasonable distribution of all seven grades. The OTE was well correlated with improvement of dyspeptic symptoms and with improvement of 5 domains of the NDI QOL scale. Responders by OTE also achieved a significantly higher proportion of days with satisfactory relief from DA-9701.

FD significantly impacts QOL. ${ }^{3}$ In this trial, All NDI score of 5 domains at the baseline were low around 50, which indicated poor QOL in patients with FD. Whereas dyspepsia score decreased after medication, the NDI score of all 5 domains significantly increased by more than 10 from baseline. Recent study suggests that a change of at least 10 points on the NDI total scale corresponds to a clinically meaningful change. ${ }^{27}$

In conclusion, DA-9701 significantly improves symptoms as well as QOL in patients with FD. DA-9701 showed non-inferior efficacy to itopride and comparable safety profile. 


\section{References}

1. Lee YY, Chua AS. What indigestion means to the malays? J Neurogastroenterol Motil 2013;19:295-300.

2. Tack J, Talley NJ, Camilleri M, et al. Functional gastroduodenal disorders. Gastroenterology 2006;130:1466-1479.

3. Choi MG, Jung HK. Health related quality of life in functional gastrointestinal disorders in Asia. J Neurogastroenterol Motil 2011;17: 245-251.

4. Lee YY, Chua AS. Investigating functional dyspepsia in Asia. J Neurogastroenterol Motil 2012;18:239-245.

5. Lee YY, Chua AS. Role of Helicobacter pylori in functional dyspepsia: more controversies than answers. J Neurogastroenterol Motil 2013; 19:417.

6. Moayyedi P, Soo S, Deeks J, et al. Eradication of Helicobacter pylori for non-ulcer dyspepsia. Cochrane Database Syst Rev 2005;(2): CD002096.

7. Lee TH, Choi JJ, Kim DH, et al. Gastroprokinetic effects of DA-9701, a new prokinetic agent formulated with Pharbitis Semen and Corydalis Tuber. Phytomedicine 2008;15:836-843.

8. Lee TH, Son M, Kim SY. Effects of corydaline from Corydalis tuber on gastric motor function in an animal model. Biol Pharm Bull 2010;33:958-962.

9. Kim ER, Min BH, Lee SO, Lee TH, Son M, Rhee PL. Effects of DA-9701, a novel prokinetic agent, on gastric accommodation in conscious dogs. J Gastroenterol Hepatol 2012;27:766-772.

10. Choi S, Choi JJ, Jun JY, et al. Induction of pacemaker currents by DA-9701, a prokinetic agent, in interstitial cells of Cajal from murine small intestine. Mol Cells 2009;27:307-312.

11. Cho YK, Choi MG, Kim SH, et al. [The effect of mosapride on quality of life in functional dyspepsia.] Korean $\mathrm{J}$ Gastroenterol 2004;43:160-167. [Korean].

12. Talley NJ, Verlinden M, Jones M. Validity of a new quality of life scale for functional dyspepsia: a United States multicenter trial of the Nepean Dyspepsia Index. Am J Gastroenterol 1999;94:2390-2397.

13. Talley NJ, Haque M, Wyeth JW, et al. Development of a new dyspepsia impact scale: the Nepean Dyspepsia Index. Aliment Pharmacol Ther 1999;13:225-235.

14. Cho YK, Choi MG, Kim SH, et al. The effect of mosapride on quality of life in functional dyspepsia. Korean J Gastroenterol 2004;43: 160-167.
15. Takahashi N, Yokota T, Murofushi N, Tamura S. Structures of Gibberellins $A_{26}$ and $A_{27}$ in immature seeds of Pharbitis nil. Tetrahedron Lett 1969;10:2077-2080.

16. Lee SP, Lee KN, Lee OY, et al. Effects of DA-9701, a novel prokinetic agent, on phosphorylated extracellular signal-regulated kinase expression in the dorsal root ganglion and spinal cord induced by colorectal distension in rats. Gut Liver 2014;8:140-147.

17. Lee TH, Kim KH, Lee SO, Lee KR, Son M, Jin M. Tetrahydroberberine, an isoquinoline alkaloid isolated from corydalis tuber, enhances gastrointestinal motor function. J Pharmacol Exp Ther 2011; 338:917-924.

18. Oh KH, Nam Y, Jeong JH, Kim IK, Sohn UD. The effect of DA-9701 on 5-hydroxytryptamine-induced contraction of feline esophageal smooth muscle cells. Molecules 2014;19:5135-5149.

19. Min YW, Ko EJ, Lee JY, et al. Nitrergic pathway is the major mechanism for the effect of DA-9701 on the rat gastric fundus relaxation. J Neurogastroenterol Motil 2014;20:318-325.

20. Camilleri M, Bueno L, de Ponti F, Fioramonti J, Lydiard RB, Tack J. Pharmacological and pharmacokinetic aspects of functional gastrointestinal disorders. Gastroenterology 2006;130:1421-1434.

21. Allescher HD, Wagner H. [STW 5/Iberogast: multi-target-action for treatment of functional dyspepsia and irritable bowel syndrome.] Wien Med Wochenschr 2007;157:301-307. [German].

22. Talley NJ, Tack J, Ptak T, Gupta R, Giguère M. Itopride in functional dyspepsia: results of two phase III multicentre, randomised, double-blind, placebo-controlled trials. Gut 2008;57:740-746.

23. Ang D, Talley NJ, Simren M, Janssen P, Boeckxstaens G, Tack J. Review article: endpoints used in functional dyspepsia drug therapy trials. Aliment Pharmacol Ther 2011;33:634-649.

24. Design of Treatment Trials Committee, Irvine EJ, Whitehead WE, et al. Design of treatment trials for functional gastrointestinal disorders. Gastroenterology 2006;130:1538-1551.

25. Matsueda K, Hongo M, Ushijima S, Akiho H. A long-term study of acotiamide in patients with functional dyspepsia: results from an open-label phase III trial in Japan on efficacy, safety and pattern of administration. Digestion 2011;84:261-268.

26. Chey WD, Howden CW, Tack J, Ligozio G, Earnest DL. Longterm tegaserod treatment for dysmotility-like functional dyspepsia: results of two identical 1-year cohort studies. Dig Dis Sci 2010;55: 684-697.

27. Jones M, Talley NJ. Minimum clinically important difference for the Nepean Dyspepsia Index, a validated quality of life scale for functional dyspepsia. Am J Gastroenterol 2009;104:1483-1488. 